\title{
Electron Microscopy Determination of Crystallographic Polarity of Aluminum Nitride Thin Films \\ N. Kuwano ${ }^{1 *}$, Jesbains Kaur ${ }^{2}$ \\ ${ }^{1}$ Microscopy Laboratory, MJIIT, UTM, Kuala Lumpur Malaysia \\ ${ }^{2}$ Research Centre for Nano-Materials and Energy Technology, Sunway University, Petaling Jaya, Malaysia \\ *kuwano.kl@utm.my
}

Keywords: electron microscopy, crystallographic polarity, wurtzite structure.

Aluminum nitride (AlN) crystallizes usually in the wurtzite structure ( $\mathrm{P}_{3} \mathrm{mc}$ ) which does not have inversion symmetry so that it has a crystallographic polarity. In this work, the polarity structure in AlN was characterized by using several methods of electron microscopy in order to examine their applicability. AlN was deposited by metalorganic vapor phase epitaxy, followed by annealing at $1550^{\circ} \mathrm{C}$. In the AlN layer, a single zig-zag inversion boundary ran transversely. Transmission electron microscopy (TEM) samples were prepared by using a focused ion beam (FIB) mill. Observation was performed with JEM-ARM200F and FEI Titan Cubed G2 microscopes at Kyushu University (Japan), and the following results were obtained. (1) Scanning TEM (STEM) High-Angle Annular Dark Field (HAADF): Even when atomic column images of $\mathrm{Al}$ and $\mathrm{N}$ are not resolved from each other, the polarity can be determined from the shape of atomic column images. (2) Scanning moire fringe imaging: When there is an inversion domain boundary, the fringe pattern can indicate its position and determine the direction of polarity. (3) Convergent beam electron diffraction (CBED): CBED is applicable for AlN under the acceleration voltage of $120 \mathrm{kV}$. Computer simulation for CBED patterns was also performed for the applicability in determination of polarity.
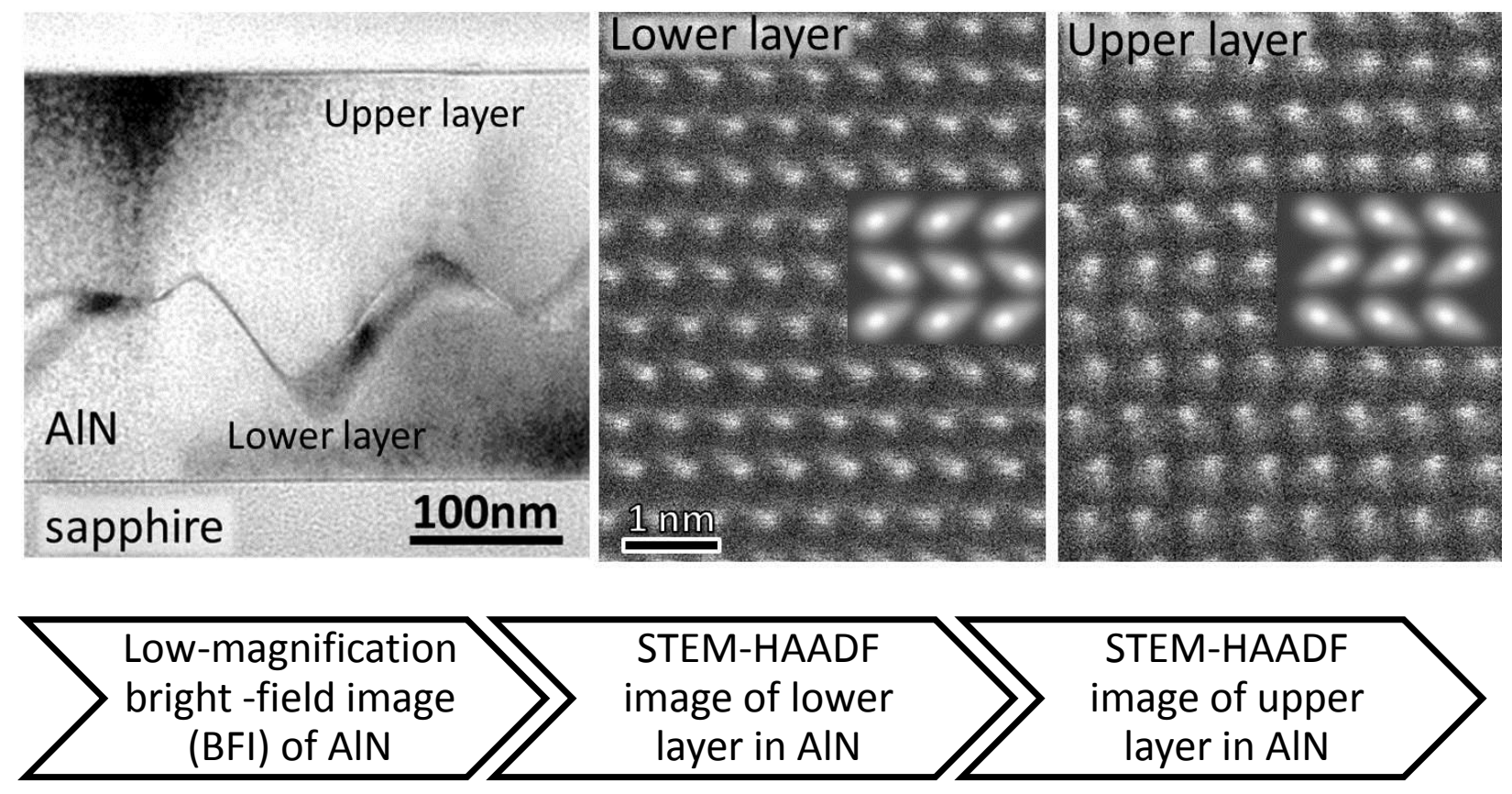